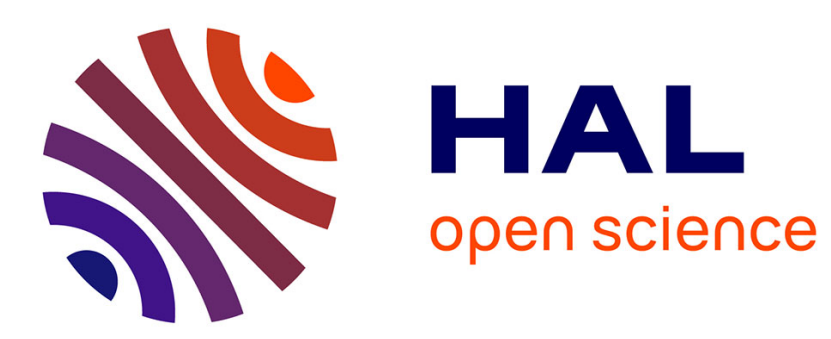

\title{
A dependable and lightweight trust proliferation approach for the collaborative IoT systems
}

Hayet Benkerrou, Mawloud Omar, Fatah Bouchebbah, Younes Ait Mouhoub

\section{To cite this version:}

Hayet Benkerrou, Mawloud Omar, Fatah Bouchebbah, Younes Ait Mouhoub. A dependable and lightweight trust proliferation approach for the collaborative IoT systems. INDERSCIENCE International Journal of Internet Technology and Secured Transactions., 2018. hal-03033579

\section{HAL Id: hal-03033579 \\ https://hal.science/hal-03033579}

Submitted on 1 Dec 2020

HAL is a multi-disciplinary open access archive for the deposit and dissemination of scientific research documents, whether they are published or not. The documents may come from teaching and research institutions in France or abroad, or from public or private research centers.
L'archive ouverte pluridisciplinaire HAL, est destinée au dépôt et à la diffusion de documents scientifiques de niveau recherche, publiés ou non, émanant des établissements d'enseignement et de recherche français ou étrangers, des laboratoires publics ou privés. 


\title{
A dependable and lightweight trust proliferation approach for the collaborative IoT systems
}

\section{Hayet Benkerrou}

Laboratoire d'Informatique Médicale, Faculté des Sciences Exactes Université de Bejaia, 06000 Bejaia, Algérie.

\section{Mawloud Omar}

Laboratoire d'Informatique Médicale, Faculté des Sciences Exactes Université de Bejaia, 06000 Bejaia, Algérie.

\section{Fatah Bouchebbah}

Laboratoire d'Informatique Médicale, Faculté des Sciences Exactes Université de Bejaia, 06000 Bejaia, Algérie.

\section{Younes Ait Mouhoub}

Laboratoire d'Informatique Médicale, Faculté des Sciences Exactes Université de Bejaia, 06000 Bejaia, Algérie.

\begin{abstract}
Trust and reputation evaluation in the Internet of Things (IoT) constitutes nowadays a major challenge that is still attracting the research community to work on and investigate. The IoT with its widely heterogeneous objects requires collaborative computation to perform heavy cryptographic operations in the most constrained resource devices. Indeed, the effectiveness of the trust and reputation assessment plays an important role for selecting the best collaborators. In this paper, we propose a dependable and lightweight trust proliferation approach for the collaborative IoT systems. By recording the malicious tasks which could be performed during the service execution, our approach introduces a new derivation of direct trust. Furthermore, it combines dynamically the direct and indirect trust evaluation to ensure more trustworthiness and reliability in the selection of the best collaborator objects. Through the simulations, we show that our trust derivation approach achieves high accuracy in the selection of efficient and confident collaborators.
\end{abstract}

Keywords: Internet of Things; Trust; Reputation; Security; Collaboration.

\section{Biographical notes:}

Hayet Benkerrou is a $\mathrm{PhD}$ student and a member of LIMED laboratory (Laboratoire d'Informatique MEDical) in the Computer Science Department, university of Bejaia (Algeria). She received the Licence diploma in Computer Science and Master degree in networks and distributed systems from the same university, in 2011 and 2013 respectively. Her research interests are mainly focused on context-awareness-based security and social relationships in Internet of Things. 


\section{H. Benkerrou et al.}

Mawloud Omar is assistant professor in the university of Bejaia (Algeria), where is member of the laboratory LIMED (Laboratoire d'Informatique MEDical) and leader of the "Networking for Healthcare Applications" group. He got his University Habilitation, $\mathrm{PhD}$ and Magister degrees in computer science from the university of Bejaia in 2014, 2011 and 2007, respectively. He got his engineer diploma in computer science from the university of Chlef (Algeria) in 2004. He was leader of the computer science department in the university of Bejaia from march 2012 to december 2015. His research activities revolve around security in computer science and network reliability, in particular the security problems of hardware, software, systems and networks. He is looking to the challenging issues related to the mobile networks, wireless sensor networks, wireless body area networks, social networks, vehicular networks, smart cities, quantum networks, the Internet of things and Big Data.

Fatah Bouchebbah is a $\mathrm{PhD}$ student and a member of LIMED laboratory (Laboratoire d'Informatique MEDical) in the Computer Science Department, university of Bejaia (Algeria). He received the Licence diploma in Computer Science and Master degree in networks and distributed systems from the same university, in 2013 and 2015 respectively. His research interests focus on security and trust models in Internet of Things.

Younes Ait Mouhoub got his Licence diploma in Computer Science from the University of Bejaia (Algeria) and Master degree in networks and distributed systems from the same university in 2013 and 2015 respectively. His research activities consist of security in Internet of Things.

\section{Introduction}

One of the most fascinating trends today is the emergence of low-cost micro-controllers that are sufficiently powerful to connect to the Internet. They are the key to the Internet of Things (IoT), where all kinds of devices become the Internet's interface to the physical world (7). The IoT (1) is an integrated part of future Internet, which is defined as a dynamic global network infrastructure with self configuring capabilities based on standard and interoperable communication protocols where physical and virtual things have identities, physical attributes and virtual personalities, and use intelligent interfaces, and are seamlessly integrated into the information network (11).

The IoT incorporates a range of technologies, which form a complex network. Due to the extremely heterogeneous devices, the IoT network contains both high and less constrained resource objects. The objects in such environment are often constrained to take in charge services requiring a performance capacity exceeding their proper ressources. Therefore, a mechanism should be based on reliable measurement in the selection of objects deemed capable of integrating operational groups to contribute to the service. Possible openings to this problem, a track that we consider interesting is to develop a trust model to define "what purpose trusts which object and how?". Furthermore, confidence is influenced by several measurable and non-measurable factors, which presents a problem of uncertainty about the value obtained during its derivation. In this case, the collaborative aspect could be introduced where trust management will be based on the reputation of the object by combining several parameters such as the capacity (hardware and/or software), the history, the honesty, etc. Several cooperative approaches have been proposed to achieve different types of services, for example routing (16), radio tasks (14), (13), security (17) (9), etc. 
The collaborative approaches are subject of threats when objects are selected without attention. A collaborator object may behave selfishly by declining the cooperation in order to save its resources or to provide incorrect information to disturb the executed service. The trust and reputation evaluation criteria remains the primary concern for measuring the selection quality. Ben-Saied et al. (8) have proved that their solution based on the cited criteria performs more efficiency in heavy computations. This collaboration increases the lifetime of the constrained resource objects. However, they don't consider any verification of the returned result by the cooperative object. This is due the fact that every object without a selfish behavior is considered as a honest regardless the inaccuracy of the provided result and the accompanying malicious tasks (for example, illegal copy, illegal transmission, etc.). In this paper, we propose a dependable and lightweight trust proliferation approach for the collaborative IoT systems, which is an improvement of (8). Our trust model relies on negative observations recorded on the collaborators in order to enhance the cooperative operations taking place in the IoT objects. Our approach focuses on a dynamic hybrid trust (direct and indirect trust combination) and proceeds in four phases to ensure a greater accuracy in the trust and reputation computation process. This precision is due to the possibility of estimating the time and the resources consumed by a malicious task and then, allowing a reliable assessment of the result returned by the cooperative object. Unlike (8), which consider each returned result as a valid value, our approach provides better performances and a high precision to select the best collaborators. To analysis the robustness, we model our trust system by the unequal probability sampling method (10) in order to get the inclusion and exclusion probabilities of honest and malicious objects from the set of the collaborators. After that, we perform extensive simulations under varying the number of executed services. The simulation results show the performance of our trust model in selecting good collaborators and discarding the bad ones, and demonstrate clearly that our solution is sufficiently resistant to the both collaborator threats and witness object threat, such as bad mouthing and on-off threat.

The remainder of this paper is organized as follows. In Section 2, we present the related work on trust and reputation evaluation in the IoT. In Section 3, we present the motivations and the system model. In Section 4 we present the detailed description of our system. In Section 5, we evaluate the performances of our system under the unequal probability sampling model, followed by the simulation results comparing the different probabilities of inclusion and exclusion of honest and malicious objects. Finally, in Section 6 , we conclude the paper.

\section{Related work}

In this section, we review from the literature the relevant approches dealing with the trust and reputation in the framework of the IoT. The main purpose of the existing solutions is to enhance the derivation precision of trust and reputation metrics.

In (4), Bossi et al. have proposed a multidimensional reputation network for service composition in the IoT. The reputation of a service provider $P_{f}$ is computed by another service provider $P_{i}$ using a multi-graph $G$ where every bow $e$ is determined by a weight $r(e)$ and a label $s$, which represents a service. $P_{i}$ computes the shortest path that connects it to $P_{f}$ by considering only the small values of the new weights. The $P_{f}$ reputation is the minimal value of a bow appearing in the shortest path using an "interchangeability function". In 
this solution, the scalability is not ensured when the size of the multi-graph $G$ increases in highly constrained memory objects.

In (12), Gong et al. have proposed a trust model combining the reputation and credential, which contains three modules: reputation module, competence study module and integration module. The reputation module computes the global score of the reputation, denoted by $\mathrm{I}(u, v)$, using a Bayesian approach, where $u$ and $v$ represent, respectively, the evaluated object and the corresponding recommandation. The competence study module computes the trust value of the objects having a recommendation $v$ and represents the latter with a vector $\left(g_{i}(v), b_{i}(v)\right)$ characterizing the good and bad behaviors, respectively. The integration module combines the trust and reputation to get the global trust value $\mathrm{I}(u, v)$ of the object. The Bayesian approach could provide a good theoretical support in the reputation computation, but it is expensive in energy consumption. Constrained objects have a greater need to collaborate with other objects in order to establish communications or to solve coverage and packet delivery problems. For these reasons, multiple cooperative techniques have been proposed for many networking services in the field of modern wireless communications.

In (8), Ben-Saied et al. have proposed a context-aware and multi-service approach for the IoT wherein the main purpose is to avoid malicious collaborators by estimating the indirect trust of each object. The trust derivation takes into account the ability of an assistant object to perform a given task in terms of resource capacities and service type. Nevertheless, this solution allows only the detection of a selfish behavior and no method is considered to verify the reliability of the results returned by the collaborator objects.

In (3), Duan et al. have proposed a trust derivation model based on the game theory. The direct reputation is computed by the watchdog technique and malicious objects are detected by the attempt of saving energy in selfish behavior. The indirect reputation is computed by the recommendation technique. To collect these recommendations while avoiding the network flooding, this approach determines a minimal mandatory number of the required recommendations to compute the indirect reputation. Indeed, a neighbor object has the choice to respond to the recommendation request or not according to its battery state. The drawback of this approach is when the rate representing the probability of response is reduced, the accuracy of the trust derivation will be decreased.

In (6), Mahalle et al. have proposed a fuzzy approach for trust-based access control in the IoT. The trust derivation is based on three metrics namely, the experiences, knowledges and recommendations. The trust value of an object $A$ to $B$ related to the experience value is computed as the ratio of the number of successful interactions regarding the number of all interactions. Direct knowledge and indirect knowledge are used to compute trust value related to the knowledges. The recommendation value is computed by the sum of those recorded in the other objects about $B$. The fuzzy trust value belongs to the interval of $[-1,1]$, which is divided by every linguistic variable into three parts affected to three linguistic values with the meaning of low, average and high trust. To obtain the final trust score, a set of inference rules are used exploiting the combination of the three results of trust reached by the experiences, knowledges and recommendations. The representation of the trust interval with linguistic values creates ambiguity when the membership function returns the same degree for two or more different classes. Moreover, fuzzy values constituting a class may vary from an application to another according to the importance of interactions. In (5), Gu et al. have proposed another variation of a fuzzy-based trust management approach for the IoT with a new architecture of physical, network and application layers. The global reputation 
value of an object is computed by the combination of three partial values computed in each layer through the fuzzy logic technique.

In (2), Chen et al. have proposed a trust management for service composition in the SOA-based IoT systems. An user performs the trust evaluation according to its direct previous satisfaction experiences and trust feedbacks from other users sharing the similar social interests. In order to measure the social similarity and filtering the trust feedbacks, this approach considers three social relationships namely, friendship, social contact, and community of interest. Indeed, this solution develops an adaptive filtering technique. Withe the latter, the best way to combine direct trust and indirect trust feedback can be achieved dynamically. Moreover, it allows each object to adaptively select its best trust parameter to minimize the convergence time and trust bias.

\section{Motivations and system model}

The IoT is a mixture of several technologies constituting heterogeneous networks, where the objects of a such environment are often dedicated to perform different services. However, the achievement of some services requires a lot of resources which are not available in highly constrained objects. For this reason, collaborative approaches are requested. The principal idea of this technique consists of selecting a set of objects helping each other to achieve complex services. Therefore, selecting the best collaborators is an important step before launching the cooperative process.

\subsection{Motivations}

Trust and reputation evaluation is one of the most pertinent tools used to ensure a good selection of the cooperative objects, as done in the system proposed by Ben-Saied et al. (8). Nevertheless, the trust in this model concerns only the ability of an object to perform an assigned service, which is inadequate because, the reliability of the executed service is not considered, the reason why it is vulnerable to several threats:

- Threats posed by a service requester: the reports sent by the requester object to evaluate its collaborators are used to compute the indirect trust of these objects. Moreover, the reports could be falsified for a malicious usage such as decreasing the indirect trust of a honest object or increasing it for a malicious one. Furthermore, the requester object is not able to check the reliability of the returned result. Its reports are about the collaborators couldn't be sufficiently reliable to compute the trust value. Indeed, when the requester becomes a witness for the future services, it will lead several bad mouthing and on-off threats.

- Threats posed by a collaborator: once the weak object (requester object) distributes the tasks related to its service, it loses the control on the latter and the collaborator object becomes the only master of the information it possesses. This privilege enables the assistant object to lead active or passive threats on the information it contains and the results of their processing. The collaborator can keep an illegal copy of the result, perform an illegal transmission of the task or its result to another object or falsify the effectuated computations.

The aim of our work is to propose an efficient strategy to select performing and trustworthy collaborator objects by monitoring their behaviors during the service 


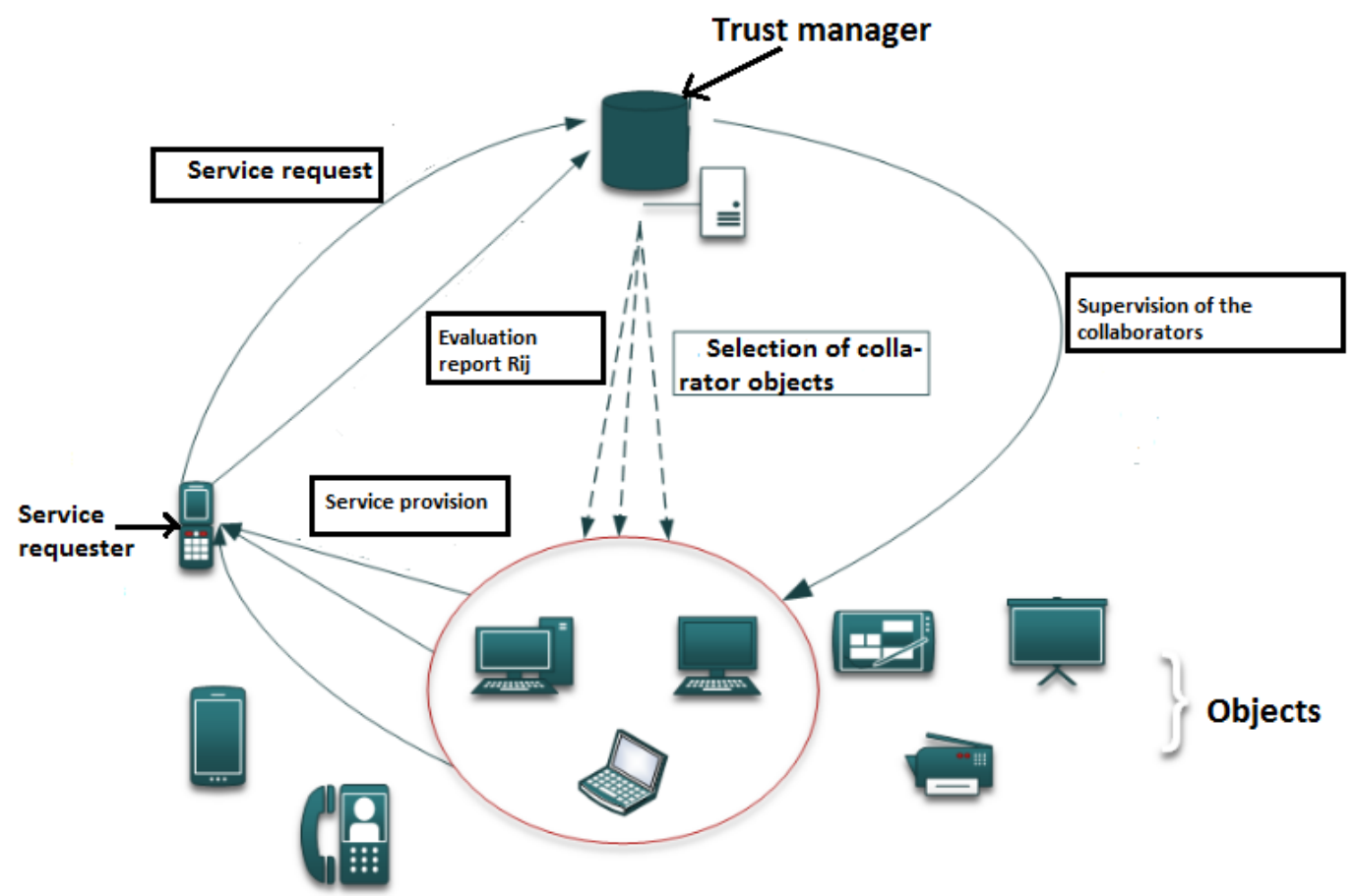

Figure 1 System model

achievement. This supervision is done by the trust manager, which facilitates the detection of the passive and active threats taking place in the collaborator objects. The direct observation covers the lack of information in the report delivered by the requester and prevents the influence of the falsified reports on the trust computation. By this way, the risk of the bad mouthing and on-off threats when the requester object becomes a witness is reduced. The malicious objects are discarded and the trust aspect is enhanced in the collaborative approaches.

\subsection{System model and assumptions}

Our approach is based on the usage of a trust manager, which is a centralized and trustworthy server with a sufficient capacity of computation and storage. We assume that we have a dense network composed of heterogeneous objects, randomly deployed, communicating through reliable channels and the tasks in these objects are executed sequently. A service contains a limited number of tasks and each task is assigned to one and only one collaborator object. The trust manager is supposed being able to estimate the percentage of the resources and the time having to be consumed by a given task and the percentage really consumed by a collaborator for executing the same task. Our approach aims to establish a community of competent and trustworthy objects helping each other to implement a set of operations related to a requested service. Figure 1 illustrates the system model of our solution.

When a constrained object requires assistance for a service, it sends a request to the trust manager. The latter checks the request and selects a set of less constrained resource objects 
collaborating with each other to assist the requester. Each collaborator object executes its task and sends the returned result to the requester. Then, the requester object expresses its satisfaction or not about the received service from each collaborator object and sends a report to the trust manager. Then, the latter either rewards (positive score) or punishes (negative score) the witness collaborator objects according to their reports. The evaluation report is a record containing the type of the performed service, the capability of a collaborator object before assisting the service, the date at which the service is obtained and the score assigned to the collaborator.

During the requested service execution, the trust manager monitors each collaborator object and records its behavior. At the end of the cooperative process, it notes the collaborators according to the recorded observations. A collaborator object having performed a suspected task or behaving selfishly obtains a negative score. A collaborator object having sent the returned result at the expected date and consuming only the expected quantity of resources is considered as a honest object and rewarded by a positive score. The recorded observations, evaluating the quality of the received service, allow the derivation of the direct trust, which is combined to the indirect trust. The set of the reports and the recorded observations will be stored in the trust manager and will be used to adjust the quality of recommendations and the reputation value of the witness objects. The used notations are presented in table 1.

\begin{tabular}{|l|l|}
\hline Parameter & Signification \\
\hline \hline$R_{i j}$ & The $j^{\text {th }}$ report evaluating the service quality provided by the object $i$ \\
\hline$W R_{i j}$ & The $R_{i j}$ 's weight \\
\hline$Q R_{j}$ & The quality of the witness object $j$ 's recommendation \\
\hline$N_{j}$ & The score given by the requester object to $i$ for evaluating the offered service \\
\hline$t_{c}$ & The current derivation date of direct trust \\
\hline$D T_{i}^{t}$ & The direct trust value of an object $i$ at $t$ \\
\hline$k$ & The number of services executed by an object \\
\hline$n$ & The number of reports considered by the indirect trust estimation \\
\hline$\alpha$ & The factor promoting of direct trust \\
\hline$\beta$ & The factor promoting of indirect trust \\
\hline$N^{F j}$ & The score of the collaborator given by the witness $F_{j}$ \\
\hline$Q R_{F j}$ & The witness object $F_{j}$ 's quality of recommendation \\
\hline$C_{j}$ & The score $N_{j}$ 's weight \\
\hline
\end{tabular}

Table 1 Notations

\section{The proposed system}

Our system combines dynamically the direct and indirect trust. During the bootstrapping phase, the trust manager discovers the network and induces artificial interactions between objects, in order to rate their trustworthiness and detect the failure objects. An object is considered as a failure if it doesn't return the result of the artificial transaction, which 
has been assigned to it. The rest is executed in three phases, namely the the selection of collaborator objects, the transaction and evaluation, and finally the learning. Il the following subsections, we present each of these phases.

\subsection{Collaborator selection}

In this phase, we provide an analysis of our selection process, whose objective is to discriminate competent and honest objects from malicious, selfish and incompetent ones with the highest accuracy and dynamicity. The resulting trust evaluation is made of two dynamically additive parts, namely the direct and indirect trust, contributing to isolate malicious and incompetent collaborator objects, and satisfying the requirements of several types of applications according to their dynamic combination. Indeed, this phase consists of three parts: (1) Indirect trust derivation, (2) Direct trust derivation, and (3) Dynamic combination of the direct and indirect trust. Before detailing our proposed direct trust evaluation and the combination process, it is worthy to give at first a brief overview of the trust management system to which our approach refers for the derivation of the indirect trust function. This trust management system is a context-aware and multi-service approach (8). Upon receiving a request from the object asking for assistance, the trust manager starts the selection process to return a set of assistant objects according to the requirements of the requested service. After that, the set of the collected reports belonging to the previous selection is restrained by computing the contextual distance $d_{i j}$ reflecting the context similarity between the old report and the target one. The most closer reports to the report of the service in question are the most meaningful reports to evaluate the indirect trust level of an object. Then, a weight $W R_{i j}$ is computed for each retained report $R_{i j}$ in order to favorite the most recent reports having the smallest contextual distance $d_{i j}$. By using the previous parameters, the scores $N_{j}$ and the quality of recommendation $Q R_{j}$ of the witness objects, one computes the indirect trust value of an object $i$ as $(8)$ :

$$
\mathrm{IT}_{i}=\frac{\sum_{j=1}^{n} W R_{i j} \cdot Q R_{j} \cdot N_{j}}{\sum_{j=1}^{n} W R_{i j}}
$$

The objects with the highest $\mathrm{IT}_{i}$ values have more chance to be selected as collaborators. This derivation model expresses only the indirect trust inferred by the recommendations of the witness objects. These recommendations evaluate solely the capability of an object to achieve a service. Using this model, there is no way to check the returned results validity by the collaborator objects in order to detect malicious tasks that a collaborator object may lead after realizing the task. The requester of the service rates the collaborators just for the service in question, so, the malicious collaborator objects will not be punished. Considering only indirect trust derivation makes the network vulnerable to both passive and active threats.

Our trust model oversees the behavior of each collaborator object. At the end of each service execution, the trust manager verifies the resource state and the executed time designated to perform the service. If the consumed resources and the execution time of an assistant object are conformed to the service requirements, the trust manager attributes to it the same score contained in the evaluation report $R_{i j}$, otherwise, it punishes it by a negative score. These direct observations are used to compute the direct trust derivation $\mathrm{DT}_{i}^{\mathrm{t}_{\mathrm{c}}}$ of an object $i$ at the current time $t_{c}$ as:

$$
\mathrm{DT}_{i}^{\mathrm{t}_{\mathrm{c}}}=e^{\mathrm{t}_{\mathrm{c}-1}-\mathrm{t}_{\mathrm{c}}} \cdot \mathrm{DT}_{i}^{\mathrm{t}_{\mathrm{c}-1}}+\frac{1}{\mathrm{k}} \cdot \sum_{s=1}^{\mathrm{k}} \mathrm{O}_{\mathrm{s}}^{\mathrm{TM}}(\mathfrak{i})
$$


where $e^{t_{c-1}-t_{c}}$ allows to promote the recent trust derivations.

In our direct trust derivation process, it is assigned the direct observation task to the trust manager instead of the objects because it is more performing and trustworthy. Our model allows also the detection and the punishment of the malicious collaborator objects, which is a preventive functionality to ensure trustworthy in the collaborative operation. Our approach reduces the direct trust of the malicious objects, while enhancing it for the honest ones in order to isolate the dishonest objects and prevent them from participating in future collaborations. By combining dynamically the indirect and direct trust, we derive the trust value of an object $i$ at the current time $t_{c}$ as:

$$
\mathrm{T}_{i}^{\mathrm{t}_{\mathrm{c}}}=\alpha \cdot \mathrm{DT}_{i}^{\mathrm{t}_{\mathrm{c}}}+\beta \cdot \mathrm{IT}_{\mathrm{i}}
$$

If $\alpha>\beta$, the direct trust derivation is promoted, otherwise, the indirect trust derivation is promoted. We note that $\alpha+\beta=1$, where $\alpha$ and $\beta$ are used to dynamically adjust the trust derivation depending on the importance and characteristics of the requested service. When a service requires a high level of confidentiality and honesty rather than the resource level of the object, the parameter $\alpha$ must be greater than $\beta$. When the requested service is only interested by the capacities of the collaborators, the parameter $\beta$ must be increased. This balancing provides more flexibility for being adapted to different services.

\subsection{Transaction and evaluation}

At the end of each cooperative operation, the requester object assesses the yield of all the collaborator objects and sends an evaluation report to the trust manager, inside which, it either rewards (positive score) or punishes (negative score) the object. This evaluation is stored in the trust manager locally and will be used later to compute the indirect trust. The requester of the service has no means to check the rightness of the returned results by the collaborator objects. The result sent can be incomplete or wrong if the object is malicious or selfish. To remedy to this problem, the trust manager proceeds to analyze the behavior of the collaborator by checking its resource state and the task execution time. When the resources and the time consumed by the collaborator meet the service requirement, the trust manager rewards the latter, otherwise, it will be punished. The evaluations are stored at the trust manager and used later to compute the future direct trust values.

\subsection{Learning}

This phase is executed in two steps:

- Quality of recommendation update: after receiving the evaluation report, the trust manager learns in advance the intention of each witness object and so, it can update its quality of recommendation. The witness object which gives a bad evaluation to a collaborator object receiving a good score is considered as a bad recommender and his quality of recommendation is decreased. When the evaluation of the witness about the collaborator object is the same as that of the requester, its quality of recommendation $\mathrm{QR}_{j}$ is increased.

- Reputation update: the reputation of an object expresses the opinion of the whole of objects of the network on the reliability of the latter after assisting other services. It serves to eliminate the malicious and incompetent objects by comparing the reputation 


\section{H. Benkerrou et al.}

of every object to the acceptation threshold. When the reputation is under the threshold, the concerned object is excluded from the network. Therefore, it can not ask or participates in the service achievement.

The reputation of a collaborator object is reduced because of its exhausted resources and it increases gradually when it will be reloaded in the case of not defective collaborator. Therefore, we add the observation saved by the trust manager to insure more accuracy in the selection as:

$$
\mathrm{R}_{\mathrm{i}}=\sum_{j=1}^{n} \mathrm{C}_{j} \cdot \mathrm{M}_{j}^{(\mathrm{i})}
$$

where $M_{j}^{(i)}=N^{F_{j}} \cdot Q_{F_{j}}+O_{s}^{T M}(i)-1$, if both $N^{F_{j}} \cdot Q_{F_{F_{j}}}$ and $O_{s}^{T M}$ are positives, otherwise $M_{j}^{(i)}=\left|N^{F_{j}} \cdot Q R_{F_{j}}\right|-\left|O_{s}^{T M}(i)\right|-1$. The parameter $M_{j}^{(i)}$ reduces the reputation of the object $i$ which has obtained a negative observation from the trust manager or a negative score from the report of the requester on the same service. Otherwise, the reputation value of the object $i$ is enhanced.

\section{Performance evaluation}

In this section, we evaluate the performances of our solution with comparison to the approach proposed by Ben-Saied et al. (8). To do this, we model the environment using the unequal probability sampling model in order to estimate the inclusion and exclusion probabilities of honest and malicious objects when selecting the collaborators. Then, we perform simulations in function of the executed service number and the hybridization factors $\alpha$ and $\beta$.

\subsection{Unequal probability sampling model}

The sampling methods (15) aim to pull samples in a concrete population intended for estimating parameters of interest with a best possible accuracy. To avoid querying two times the same entity, real samplings focusing on finite populations are performed by drawing without replacement. The samples are no longer consisting of independent variables and the draw is not always done with the same probabilities.

Let's consider $\mathrm{U}=\{1,2, \cdots, \mathrm{N}\}$ a population of entities also called database poll, where $\mathrm{N}$ represents the population size. We note $\mathrm{Y}$ the variable of interest whose values are $\left\{Y_{1}, Y_{2}, \cdots, Y_{n}\right\}$. We assume that $Y_{i}$ is obtained without errors if the entity $i$ is selected. A sample is a subset of $n$ entities of the population and $\tau=\frac{n}{N}$ represents the sampling rate. There is $C_{N}^{n}$ possibilities of separated samples, each one is noted by $s$. In a random sampling, each entity $i$ of the population has a drawing probability, where the well defined inclusion probability $\Pi_{i} \neq 0$ to allow the estimation without bias. We note that the sum of probabilities of inclusion is equal to $\sum_{i=1}^{N} \Pi_{i}=n$ and $\Pi_{i}$ is equal to the amount of probabilities of the samples containing the entity $i: \Pi_{i}=\sum_{s(i \in s)} P(s)$. A plan of poll is a probability distribution on the set of samples. We consider two cases (10): (1) the simple random sampling, which constitutes the basis of the other methods. It is an equiprobable draw without discount. We have, therefore, $\Pi_{i}=\frac{n}{N}$ and all samples $C_{N}^{n}$ are equiprobable; (2) the sampling with unequal probabilities, in which we have an auxiliary variable $x_{i}>0$, 
such as $i \in U$, enough proportional to the variable $Y_{i}$. We select the units with unequal probabilities proportional to the $x_{i}$. To do this, the inclusion probability is computed as:

$$
\Pi_{i}=\frac{n \cdot x_{i}}{\sum_{j=1}^{N} x_{j}}
$$

If $\Pi_{i}>1$, the corresponding entities are selected in the sample with an inclusion probability equals to 1 and $\Pi_{i}$ are recomputed according to the same formula for the remaining entities.

\subsection{The proposed model}

We model the environment using the sampling with unequal probabilities previously defined. The objective of this modeling is the analysis of the trust and the reputation values. To do this, we compute the inclusion probability of a honest object in the set of collaborators, as well as the inclusion probability of a malicious collaborator in the set of bad objects.

\subsubsection{Inclusion probabilities}

These probabilities illustrate the effectiveness of the proposed solution. Their computation process is based on the hybrid trust values, which is a decisive parameter in the selection or inclusion of the good collaborator objects. The inclusion probability computation for the first requested service is performed as follows. The sampling base is composed of $\mathrm{N}$ objects, which have initially the same value of trust. We draw a random sample (a group of collaborators) of $n$ objects in order to assist the first requester object to achieve its service. The draw is without discount because a collaborator must be included only once in the sample. Therefore, this is the subject of a simple random sampling. In this case, all the samples are equiprobable and the sampling rate is $\tau=\frac{n}{N}$. The inclusion probability computation for the next requested services is performed as follows. When the system is in activity, the objects executing services and therefore, the trust values change. Each object $i$ has a variable $T_{i}$ that represents its value of trust. To this effect, we only need to select objects with unequal probabilities proportional to $T_{i}$. However, the values $T_{i} \in \mathbb{R}$, making them unsuitable for this type of sampling which requires values in $\mathbb{N}^{*}$. Indeed, we proceed to the normalization of these values. The normalization allows the transformation of the trust values $T_{i}$ from the set $\mathbb{R}$ towards $\mathbb{N}^{*}$ while keeping the accuracy of the gap between these values. Either $T_{i}$ the trust value of an object $i$, we determine firstly the minimum of the values $T$ and we normalize these values as:

$$
\mathrm{T}_{i}^{\prime}=\left(\mathrm{T}_{\mathrm{i}}+|\min +1|\right) \cdot 10^{\omega}
$$

As is the normalized value, $\min$ is the minimum value in the set of trust values $T$ and $\omega$ is the factor of precision. Finally, the probability of selection of each object $i$ is computed as:

$$
\Pi_{i}=\frac{n \cdot T_{i}^{\prime}}{\sum_{i=1}^{N} T_{i}^{\prime}}
$$




\section{H. Benkerrou et al.}

\subsubsection{Exclusion probabilities}

Based on a population of $\mathrm{N}$ objects, we compute the inclusion probability of an object in the set of malicious objects to be excluded from the network. We assume $m$ be the number of malicious objects. So, the sample to be selected has a size of $m$ items. Each object has its own value of reputation. Therefore, the model leads to a sampling with unequal probabilities. The normalization of the reputation values is required in order to be adapted to our system, such as:

$$
R_{i}^{\prime}=\left(-R_{i}+|\max +1|\right) \cdot 10^{\omega}
$$

where $R_{i}^{\prime}$ is the new normalized reputation value of the object $i$ and max is the maximum value in the set of reputation values. The exclusion probabilities are computed by the following formula:

$$
\Pi_{i}=\frac{m \cdot R_{i}^{\prime}}{\sum_{i=1}^{N} R_{i}^{\prime}}
$$

\subsection{The model simulation and results}

We consider an environment with $\mathrm{N}=200$ heterogeneous smart devices where 100 objects are highly constrained in resources. We randomly select $10 \%$ out of all objects as malicious objects $(\mathrm{m}=10 \%)$. We initialized the quality of recommendation to 1 for each collaborative operation. The requester object selects assistant objects among the less constrained resource objects to request a service pulls randomly among six different types of services. 40 collaborator objects are selected by the system to assist the requester object in its service, and the cost of the latter is determined in a regular manner. The results are the average of 100 simulation iterations. In the following, we present the simulation results of our system, in which we compare its performances with those of the model proposed by Ben-Saied et al., which is a special case in our system $(\alpha=0$ and $\beta=1)$.

\subsubsection{Inclusion probability of a honest object}

Figure 2 shows the variation of the inclusion probability of selecting a honest collaborator according to the the number of executed services. For our solution, the probability of selecting a honest collaborator increases with the increment of the number of executed services (experiences), and it is better compared to the model of Ben-Saied et al. Our trust model considers only the honest assistant objects during the service execution because it checks the objects behavior in the direct trust derivation, which provides to them a better reward. This is the reason of the large gap between the trust values of the honest and dishonest collaborators. The selection probabilities of honest collaborators are increased, unlike the solution of Ben-Saied et al., which focuses only on the capability of an object to perform a given service. The model of Ben-Saied et al. marks all the collaborator objects in the same way, as a result, the trust values and the selection probabilities of honest objects are close-up. 


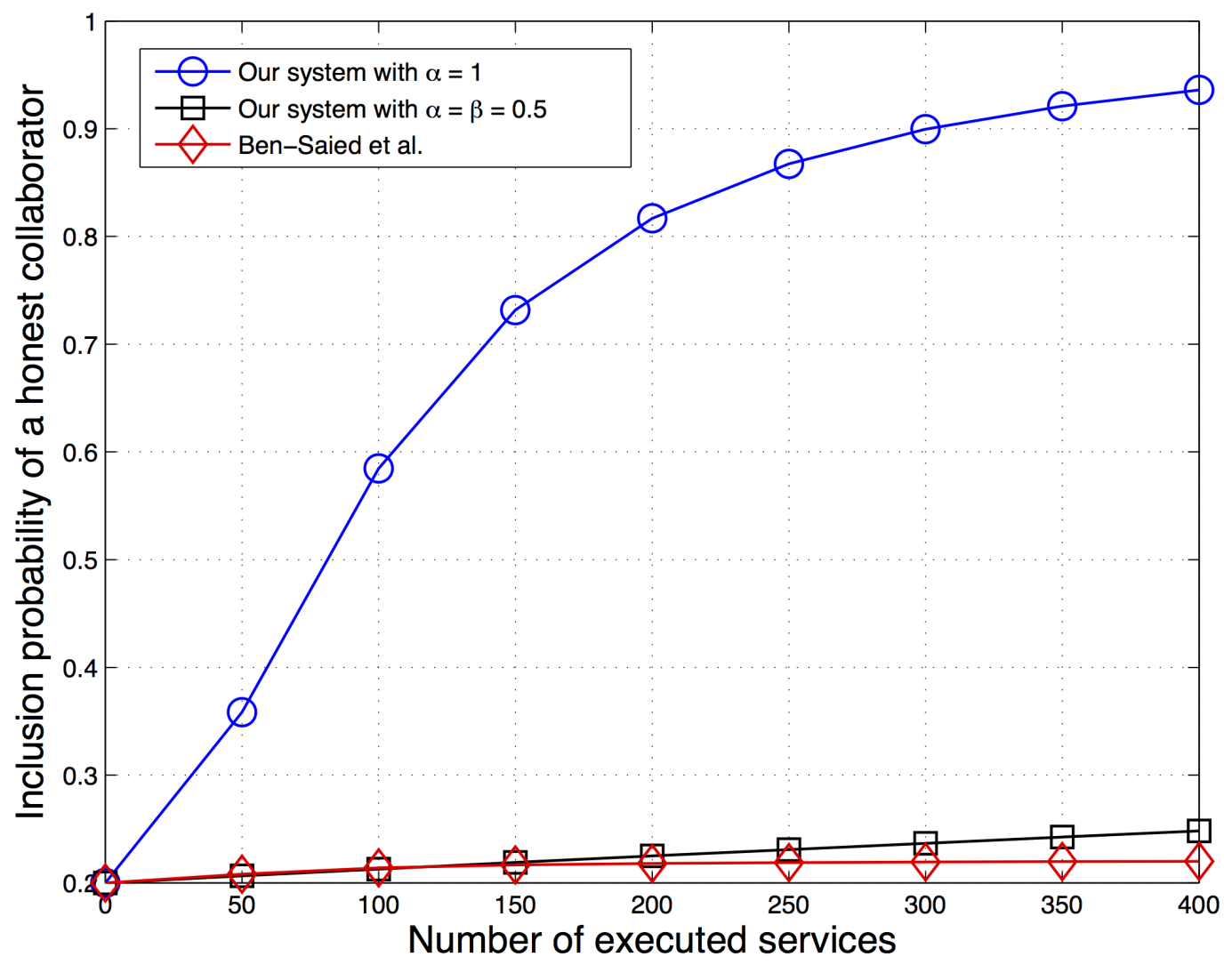

Figure 2 Inclusion probability of a honest collaborator with $\omega=2$

\subsubsection{Inclusion probability of a malicious object}

Figure 3 illustrates the evolution of the probability of selecting a malicious collaborator according to the number of executed services. We note that the probability of selecting a bad collaborator in the case of the model of Ben-Saied et al. is almost the same as that of a honest collaborator in the figure 3, because it does not mark a difference between the honest and the malicious collaborator object when it conserves a high resource level and participates in the cooperative process, every assistant object returning a result is considered as a honest object without analyzing its behavior. Unlike our system, noting an exponential fall of the inclusion probability of a malicious object. This is due to the negative observations recorded on the malicious collaborators involving their discarding from the selection and reducing their trust values, as well as their probability of inclusion in the next service assistance. We note that whenever the value of the factor $\alpha$ increases, the inclusion probability of a malicious object decreases. This is justified by the difference in the trust values of the assistant objects with the variation of $\alpha$, thus, it facilitates the distinction between the honest and dishonest collaborator objects. These results show the importance of the direct trust implied in our model. 


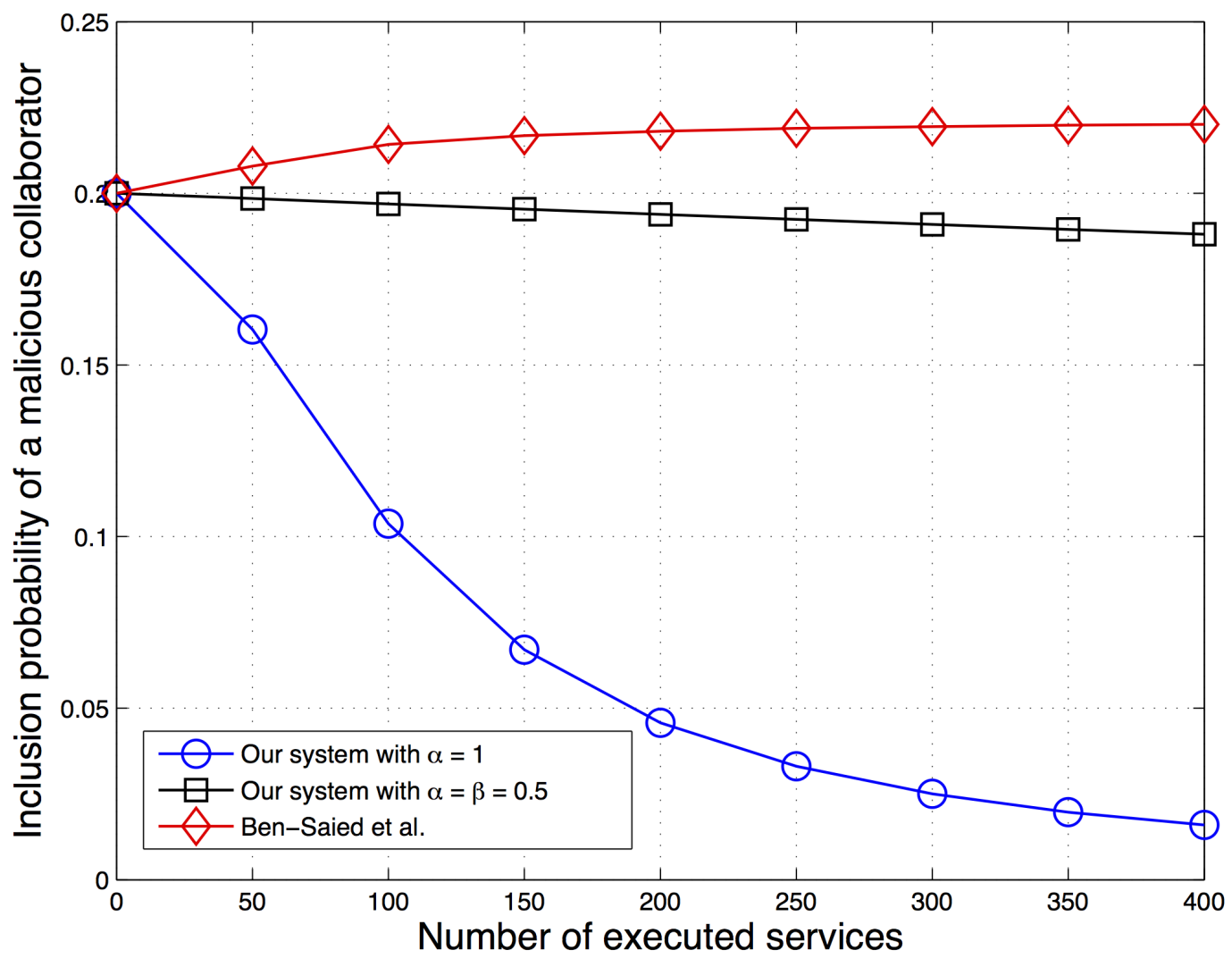

Figure 3 Inclusion probability of a malicious collaborator with $\omega=2$

\subsubsection{Exclusion probability of a honest object}

It is important to have only the best collaborator objects in the network in order to facilitate the computing of the context similarity by the contextual distance, the weight of the reports and the selection of the collaborator objects able to achieve the requested service. This precaution is taken into consideration by excluding the collaborators whose reputation values are less than a given threshold. Figure 4 illustrates the evolution of the exclusion probability of a good collaborator according to the number of executed services. We note that regardless the $\alpha$ and $\beta$ values, our model provides better results following the exclusion of the honest collaborators comparing the model of Ben-Saied et al. This is due to the recorded direct observations guaranteeing a best precision in the reputation derivation. Indeed, the positive observation recorded by the trust manager on the honest objects prevent the impact of the false negative scores given by the witness objects via their reports. Hence, we deduce from the graph that our trust model is more efficient in remedying the bad mouthing threat and the risk of confusion between a honest and dishonest collaborator is reduced.

\subsubsection{Exclusion probability of a malicious object}

Figure 5 shows the variation of the exclusion probability of a malicious collaborator object according to the number of executed services. We note that our system displays the best 


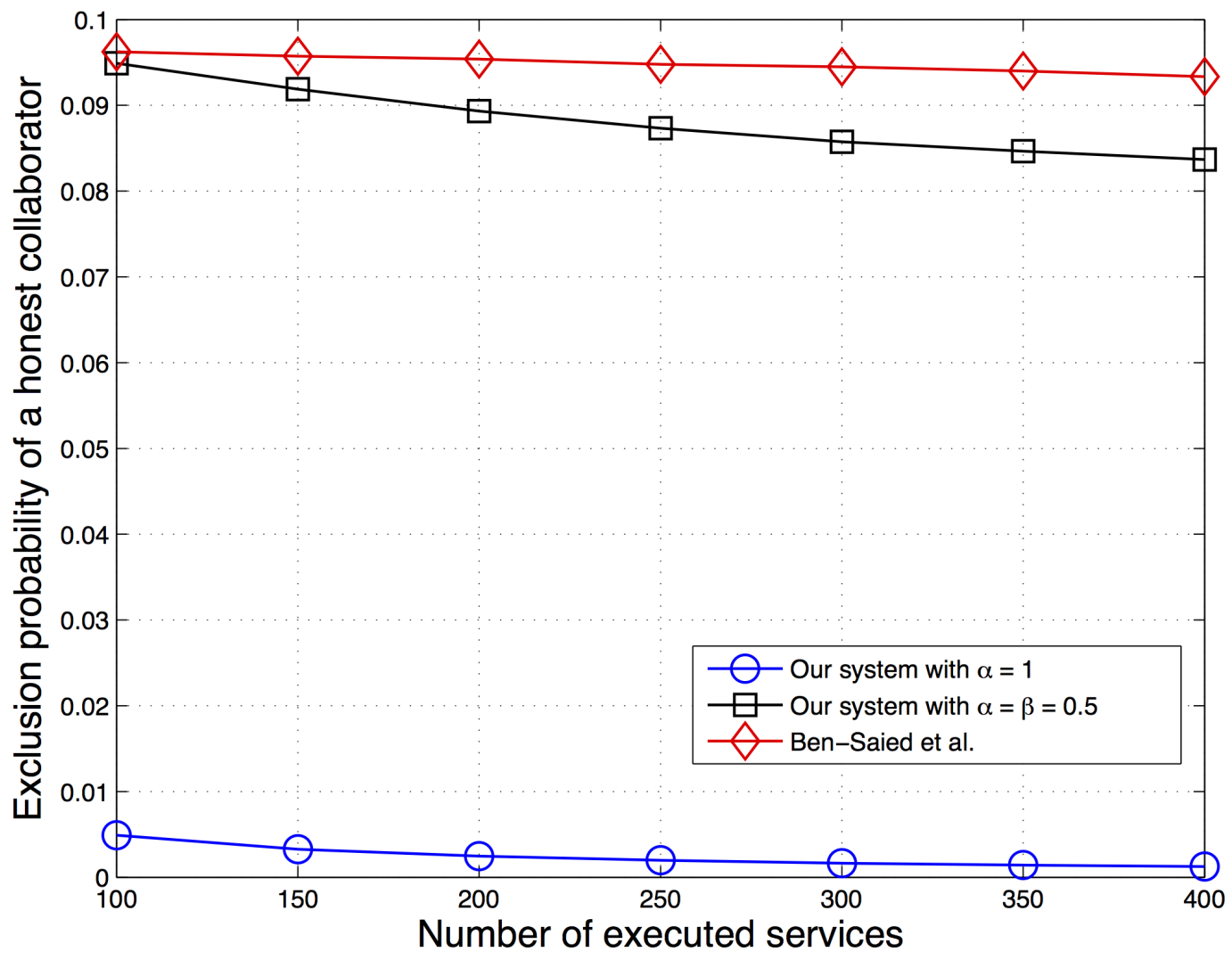

Figure 4 Exclusion probability of a good object with $\omega=2$

performance. Indeed, the exclusion probabilities of a dishonest collaborator is widely increased in our trust model than in the model of Ben-Saied et al. The results show that our reputation assessment has a good prevention of the on-off threat because, the direct observation involvement strengthens the consideration of only the reports containing a honest score.

\section{Conclusion}

In this paper, we designed a dependable and lightweight trust proliferation approach for the collaborative IoT systems for lightweight collaborative approach in the IoT. Our approach is based on the hybrid trust and reputation evaluation to select competent and honest collaborators for assisting highly constrained resource objects in the IoT. Our solution improves the trust derivation in by implying the direct trust derivation. This improvement is assured by the trust manager which supervises cooperative objects behavior in order to detect not only the selfish behavior, but also the malicious tasks that could be performed by this latter. The trust manager allows the detection of these threats by estimating two parameters: the execution time and the consumed resources. Our model is composed of two parts, namely direct and indirect trust derivation. To validate our solution, we have modeled 


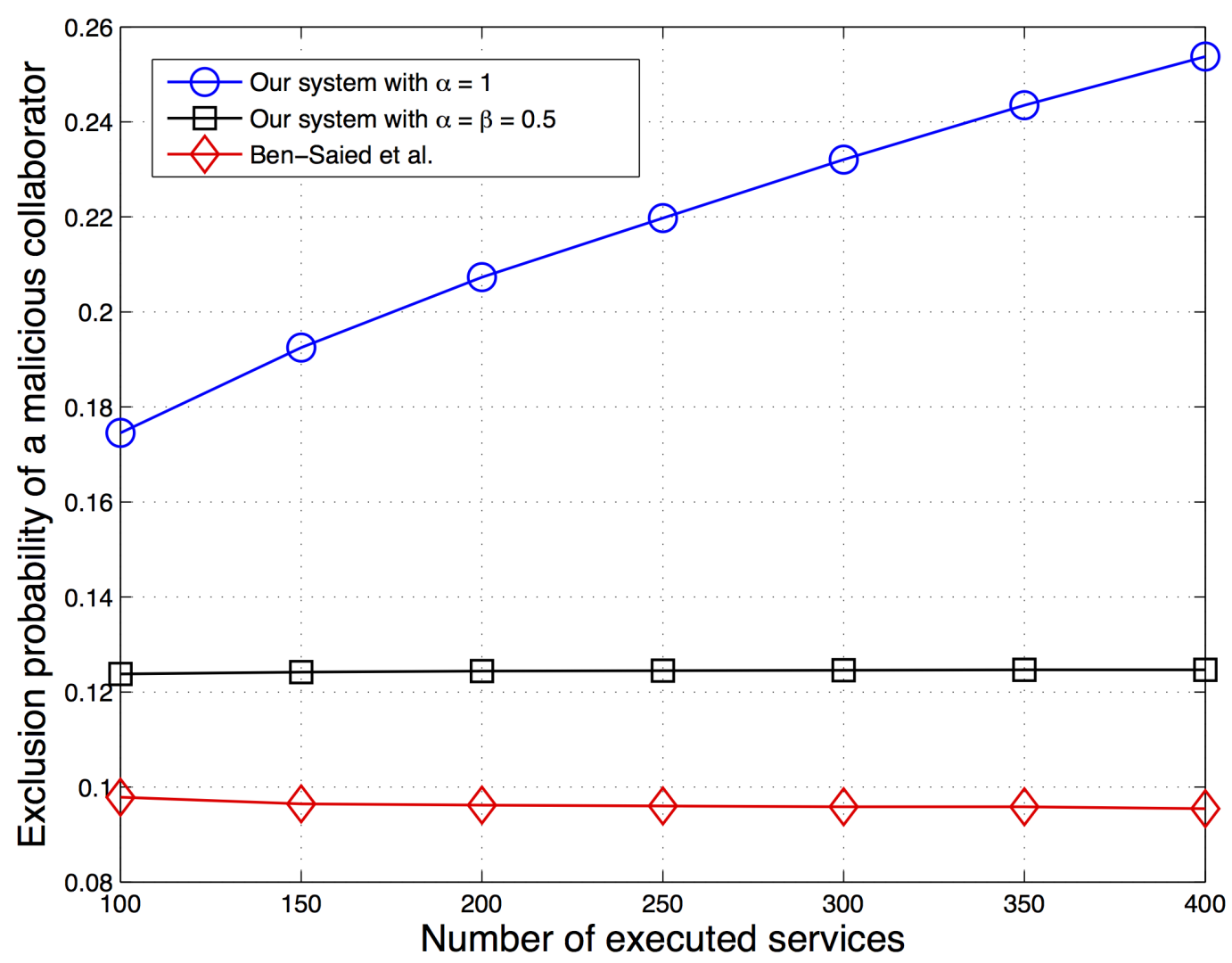

Figure 5 Exclusion probability of a bad object with $\omega=2$

it using the sampling method in order to compute the inclusion and exclusion probabilities of both good and bad objects. These probabilities are analyzed with the variation of the number of executed services by performing several simulations. The obtained results show that our system offers more opportunities to select good collaborators in terms of competence and honesty and permits the exclusion of all malicious and compromise objects.

\section{References}

[1] S-E. Bibri. The Shaping of Ambient Intelligence and the Internet of Things. Book, Springer, Atlantis Ambient and Pervasive Intelligence, Vol. 10, 2015.

[2] R. Chen, J. Guo and F. Bao. Trust Management for Service Composition in SOAbased IoT Systems. International IEEE Conference on Wireless Communications and Networking, pp. 3444-3449, Istanbul, Turkey, 2014.

[3] J. Duan, D. Gao, D. Yang, C-H. Foh and H. Chen. An Energy-aware Trust Derivation Scheme with Game Theoretic Approach in Wireless Sensor Networks for IoT Applications. IEEE Internet of Things Journal, Vol. 1, No. 1, pp. 58-69, 2014. 
[4] L. Bossi, S. Braghin and A. Trombetta. Multidimensional Reputation Network for Service Composition in the Internet of Things. In International IEEE Conference on Services Computing, pp. 685-692, 2014.

[5] L. Gu, J. Wang and B. Sun. Trust Management Mechanism for Internet of Things. China Communications, Vol. 11, No. 2, pp. 148-156, 2014.

[6] P-N. Mahalle, P-A. Thakre, N-R. Prasad and R. Prasad. A Fuzzy Approach to Trust Based Access Control in Internet of Things. Third International IEEE Conference on Wireless Communications, Vehicular Technology, Information Theory and Aerospace \& Electronic Systems, pp. 1-5, Atlantic City, NJ, 2013.

[7] Z. Sheng, S. Yang and Y. Yu. A survey on the IETF Protocol Suite for the Internet of Things: Standards, Challenges, and Opportunities. IEEE Wireless Communications, pp. 91-98, 2013.

[8] Y. Ben-Saied, A. Olivereau, D. Zeghlache and M. Laurent. Trust Management System Design for the Internet of Things: A Context-aware and Multiservice Approach. Computers \& Security, Vol. 39, pp. 351-865, 2013.

[9] Y. Ben-Saied, A. Olivereau and D. Zeghlache. Energy efficiency in M2M networks: a cooperative key establishment system. In third International congress on ultra modern telecommunications and control systems, Budapest, 2011.

[10] G. Saporta. Probabilitnalyse des Donn et Statistiques. Book, Technip, Third edition, 2011.

[11] Cluster of European Research Projects on the Internet of Things. Vision and Challenges for Realising the Internet of Things. Scientific Report, 2010.

[12] J. Gong, J. Chen, H. Deng and J. Wang. A Trust Model Combining Reputation and Credential. In International IEEE Conference on Information Engineering, pp. 635638, Taiyuan, Shanxi, 2009.

[13] Y-W. Hong, W-J. Huang, F-H. Chiu and C-C-J. Kuo. Cooperative communications resource constrained wireless networks. IEEE Signal Processing Magazine, Vol. 24, No. 3, pp. 24-47, 2007.

[14] A. Krohn, M. Beigl, C. Decker, T. Riedel, T. Zimmer and D. Garces. Increasing connectivity in wireless sensor network using cooperative transmission. In third international conference on networked sensing systems, Chicago, USA, 2006.

[15] P. Ardilly and Y. Tillxercices Corrige Modes de Sondage. Book, Ellipses Marketing, 2002.

[16] E. Royer and C-K. Tob. A review of current routing protocols for ad hoc wireless networks. IEEE Personal Communications, Vol. 6, No. 2, pp. 46-55, 1999.

[17] M. Mambo, K. Usuda and E. Okamoto. Proxy signatures: delegation of the power to sign messages. IEICE Transactions on Fundamentals of Electronics, Communications and Computer Sciences, Vol. E79-A, No. 9, pp. 1338-1354, 1996. 